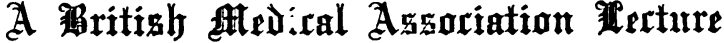
on

\section{RECENT ADVANCES IN THE SURGERY OF THE CHEST.}

Delivered before the Bradford Division, April, 1926, BY

H. S. SOUTTAR, C.B.E., M.CH., F.R.C.S., SURGION, WITH CHARGE OF OUT-PATIENTS, LONDON HOSPITAL. (With Special Plate.)

Surgical procedures in connexion with the chest differ from those in other regions of the body in the peculiar mechanical difficulties which they present. On the one hand, the chest and its contained viscera are in constant motion; on the other hand, in the pleural cavity alone of all regions of the body is there a constant negative pressure. The latter is by far the most important consideration in dealing with the thorax, since it means that unless very special precautions are taken an opening into the pleural cavity will generally be followed by the immediate collapse of the lung on that side.

This pleural suction, as it might well be called, varies between $6 \mathrm{~mm}$. of mercury in expiration and $30 \mathrm{~mm}$. on deep inspiration, but it must be remembered that on closing the glottis and coughing it may be converted into a positive pressure of $100 \mathrm{~mm}$. or more of mercury. So long as the thorax is closed the mechanical conditions are not affected by the existence of a potential pleural cavity, but the moment it is opened the conditions on the two sides of the chest become entirely different. If the opening is large the viscera on this side are subject simply to atmospheric pressure, whilst on the opposite side they are subject to pressures which may vary, as we have seen, between $-30 \mathrm{~mm}$. and $+100 \mathrm{~mm}$. of mercury.

If the two sides of the chest were separated by a rigid partition no great harm might result. It would simply mean that one side was thrown out of action so far as respiration was concerned. They are, however, separated not by a rigid partition but by a very mobile mediastinum, and, moreover, this mobile mediastinum contains the heart, the great vessels, and a mass of important nervous structures. Such variations in the pressures on its two sides, often as sudden as they are violent, will literally throw the mediastinum from side to side, producing the condition known as flapping of the mediastinum. The dangers of such a condition need scarcely be stressed, and yet it is the immediate result of a large opening made into the chest without due precautions. Such a state of affairs is of common occurrence in the ordinary treatment of acute empyema. Its recognition and the elaboration of means to avoid it form an important advance in thoracic surgery.

\section{Acote EMpyema.}

Acute empyemata occur in two forms-the adherent and the non-adherent. The former are usually pneumococcal, and will often show pneumococci in pure culture. They are often loculated and limited to one portion of the pleural cavity; they contain thick pus which can only be aspirated with difficulty; the pleural surfaces are covered with thick layers of plastic lymph, and floating in the cavity itself are large masses of soft fibrin. In direct contrast to this the non-adherent form is usually due to streptococci or to a mixed infection; it is necessarily complete, the pleural cavity is full of thin pus, and as gas-forming organisms are frequently present the condition is generally one of pyopneumothorax. In this form the intrathoracic pressure may be very high, the intercostal spaces bulge, the mediastinum is pushed over to an excessive degree, and the diaphragm and liver are pushed downwards. Obviously the two forms involve surgical and mechanical considerations of a very different nature, and they demand very different methods of treatment.

In the first or adherent form there can, in my opinion, be no doubt that the best method of treatment is to open the cavity freely, evacuate the pus, clear out all solid masses of fibrin, and drain by a closed method. The adhesions will prevent collapse of the lung and the forma- tion of a complete pneumothorax, and the free opening, by giving adequate access to the interior of the chest, makes it possible to remove the large solid masses whose presence retards sterilization and induces the formation of massive adhesions. A large tube is now inserted at the lowest point of the cavity, and the tissues are carefully closed around it, so that it fits quite airtight. A large cork or a perforated sheet of rubber is threaded tightly over the tube, and when the dressings are applied this holds the tube in place. It projects through the dressing, and it is connected to a long rubber tube the end of which hangs below fluid in a jar on the floor beside the patient's bed. As the fluid trickles down it, this exerts a slight suction which assists the expansion of the lung.

In the non-adherent form, on the other hand, a free opening into the pleural cavity is exceedingly dangerous, involving as it does gross disturbance of the equilibrium of the thorax, and producing in an acute form the "flapping mediastinum " to which we have already referred. It is far safer to relieve the pressure by repeated aspiration, until sufficient time shall have elapsed for the formation of a certain amount of protective adhesions. Once these have been formed the pleural cavity can be opened without the danger of an immediate collapse of the lung, but even then it is much better to adopt the method of closed drainage we have described. With open drainage an initial pneumothorax is inevitable, and it may lead to such thickening of the pleura as will seriously interfere with expansion of the lung, whilst there is always a grave risk of secondary infection of a large cavity. Closed drainage is quite easy to arrange-it is more comfortable for the patient, it avoids these risks, and the constant slight suction assists the lung to expand.

\section{Suppurative Bronchiectasis.}

The importance of these mechanical considerations in connexion with the treatment of suppuration in the pleural cavity will be seen. Let me now direct attention to their bearing on suppuration in the lung itself. Sooner or later this is certain to resolve itself into a suppurative bronchiectasis, and for the sake of brevity I shall consider it solely from that aspect.

Suppurative bronchiectasis may have its origin in some mechanical interference with the bronchi, or in infection of the parenchyma of the lung-as, for instance, after an unresolved pneumonia. In either event a very similar state of affairs ultimately results, for the bronchi become dilated from weakening of their walls and obstruction to the egress of air, whilst there is always a greater or less degree of destruction of the lung tissue itself. A ragged abscess cavity may be formed, into which open widely dilated bronchi; or in the more chronic cases the bronchi themselves may form the cavity in which pus collects. In both cases the patient coughs up large quantities of foul pus, and suffers from a severe degree of septic absorption. The problem for the surgeon is the draining of these cavities, and the prevention by adequate ventilation of the growth of the anaerobes for which these cavities provide such a perfect soil.

It is well, however, that we should recognize how often these conditions have a mechanical, or, unfortunately, even a surgical, origin. The commonest cause of bronchiectasis is mechanical interference with the passage of air through the bronchi, and of this one of the commonest causes is the inhalation of a foreign body. This may be any small object, such as a button, a tin-tack, or a collar stud; or it may be a fragment of bone from food the patient was eating, as in a case I shall presently describe. The frequency of such an event, and the gravity of the results, are not sufficiently recognized, but thanks to the pioneer work of Chevalier Jackson the conviction is steadily gaining ground that a large number of cases of obscure pulmonary sepsis are traceable to this source. The following is a striking example.

I was asked by Lord Dawson to see one of his patients in the London Hospital, a woman of 20 with an obscure condition in the right side of the chest. a She told me that five months previously, when eating a mutton chop, she suddenly choked, and thought that a fragment of bone had gone down the wrong way. gradually subsided, learing her with a dry cough. 
One month later she began to feel ill, her temperature rose, and her doctor, on exploring the right side of her chest, found pus. She was admitted to a hospital, where her chest was again organisms and offeny yellow pus was aspirated, containing many perature, ranging between $\mathrm{C}^{\circ}$ and $102^{\circ}$, rising on one occasion perature, ranging between $58^{\circ}$ and $102^{\circ}$, rising on one occasion ing yellow mucoid masses. Over the right lower chest the percussion note was dull, and breath sounds were absent. Under the impression that the case was one of empyema a rib was resected, the pleural cavity opened, and one ounce of pus was evacuated. This was followed by the drainage from the wound of quantities of very foul pus, but her temperature remained irregular, ranging between $96^{\circ}$ and $102^{\circ}$.

When I saw her in the London Hospital she was a very ill looking woman, coughing up enormous quantities of horrible pus. There was an open wound in the right side of the chest from which pus was pouring, but thi

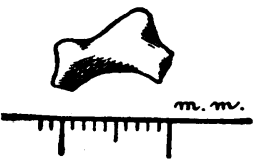
periodically closed. She felt better when it was open. The right side of the chest showed marked collapse, and there was some dorsal scoliosis with the concavity to the right. The movements of the right diminished, and the breath sounds were weak. There was increased vocal resonance and bronchophony over the right base. Repeated examinaance and bronchophony over the right base. Repeated examinasputum. A blood count showed 7,320 leucocytes, of which 70 per cent. were polymorphs. $X$-ray examination showed a cavity containing fluid in the right lung, whilst the whole of the lower portion of the right lung was opaque and the diaphragm could not be seen.

In view of the long history of the case arrangements were made for a full exploration of the chest, but bronchoscopy was first attempted. It was rendered difficult by the masses of purulent sputum which were being constantly coughed up I found the right bronchus intensely inflamed, the wall looking like red velvet, right bronchus intensely inflamed, the wall looking like red velvet, cavity was entered containing pus and debris. The thick pus was removed with some difficulty, and finally the bottom of the cavity, formed by red granulation tissue, was reached at a distance of fourteen inches from the teeth. In this granulation tissue a minute white spot could be seen, and on gradually clearing away debris it became a white ridge across the tube, which a probe revealed to be a hard rough structure. On seizing it with alligator forceps it proved to be some object larger than the tube, through which it could not be drawn. The whole instrument was therefore withdrawn, with the forceps holding what was evidently a foreign body. It proved to be a piece of mutton bone of irregular formation, about half an inch across. The immediate improvement in the patient's condition was remarkable, and though her chest has not entirely cleared and she still coughs up a little pus, she considers. herself too well for any further operation.

The aspiration of a foreign body is only too likely to occur during a surgical operation, unless the greatest care is taken to avoid it. Particles of vomited material, or plugs of mucus from the mouth, may easily reach the bronchi, and there can be no doubt that many cases of post-operative pneumonia are due to this cause, whilst fragments of teeth or of tonsils may give rise to very serious trouble. It is only recently that attention has been called to the pulmonary complications from this source which may follow tonsillectomy, but the statement of Frederick Moore that pulmonary abscess occurs once in every 3,000 tonsillectomies shows how serious a matter it may be.

The pressure of tumours outside the bronchi may give rise to bronchiectasis and the formation of a pulmonary abscess, and this frequently occurs in carcinoma involving the hilum of the lung. In a case recently referred to me by Dr. Rowlands I drained an enormous abscess of the right lung only to find post mortem that a carcinoma had destroyed the right bronchus, invaded the hilum of the left lung, and actually penetrated into the left auricle of the heart.

The diagnosis of bronchiectasis is the realm of the physician, but the average case can unfortunately be diagnosed from very obvious symptoms, by the large amount of pus which the patient coughs up, and by the horrible fetor which distinguishes it. Naturally there is some febrile disturbance, often an irregular swinging temperature, and the patient is ill. But there is not as a rule marked emaciation, and the disease may pursue its course over a long period, whilst the patient is a misery to himself and a terror to his friends. The $x$-ray appearances are fairly characteristic. There are dense opacities due to infiltrated and fibrotic lung and to bronchi filled with pus, and from these opaque areas shadows radiate out towards the periphery. Occasionally an area is seen, roughly circular in outline, the upper portion transparent, the lower filled by a dense shadow with a horizontal upper margin. If, on moving the patient, this margin is seen to remain horizontal, moving relatively to the patient, it is evident that we are looking at a cavity containing fluid, a true abscess cavity in the lung. In a typical case the picture contrasts with that of empyema, since the opacity is surrounded by a zone of more transparent lung.

The outlook for these cases apart from surgical intervention is poor. It is true that they may be taught to empty the cavities by postural methods, and that under careful hygiene they may improve. But in most cases, in spite - of remissions, the progress is steadily downward, continued sepsis produces its inevitable results, and at any time acute haemoptysis, a secondary empyema, or a cerebral abscess may occur. Until a few years ago it was thought that little could be done for these cases by surgical means, but new methods seem to point to a much more hopeful future.

It is understood that every effort has been made to determine the exact situation and extent of the disease, and that the possibility of a foreign body, or perhaps the foreign body itself, has been eliminated. The next procedure is probably a full surgical exploration of the chest. Under a combination of regional and intratracheal anaesthesia an incision is made in the seventh intercostal space, and unless dense adhesions are encountered it is extended through almost the whole length of that space. A rib spreader is now inserted and the ribs separated to a distance of perhaps four inches. In the absence of adhesions a full view of the lung can now be obtained, it can be fully palpated, and its condition can be completely investigated.

In general, unless an aspirating needle has been used and the pleura infected, the lung will be found free of adhesions. The affected lobe will be found to be somewhat rigid from infiltration, and it will often be of a darker colour than the normal lung. If the air pressure in the intratracheal tube is reduced the normal lung shrinks at once, but the diseased lung only to a less extent. It must be remembered that it forms a spongework, with dilated bronchi filled with pus and prevented from collapsing by the infiltration of their walls, and this conception must be borne in mind in considering the problem of treatment. Occasionally the mero opening of the thorax and the letting in of air may produce a sufficient degree of collapse in the diseased lung to effect a cure. But this must be a rare event, and can only occur in early cases where the degree of infiltration is slight.

The obvious surgical course. is to drain away the pus, but here we are faced by two serious difficulties. In the first place, if we incise the lung we inevitably infect the whole of a widely open pleural cavity, and we produce a septic complete empyema which communicates with the bronchi, making matters ten times worse. In the second place, you cannot drain a bronchiectatic lung by simply incising it. As Lilienthal neatly puts it, there is a great difference between emptying a vessel by knocking a hole in its bottom and trying to get the liquid out of a water-soaked sponge by incising it. So much, indeed, is Lilienthal impressed by this difficulty that he advocates complete excision of the affected lobe. In his hands this drastic procedure has given wonderful results, though even he finds a mortality of nearly 50 per cent. The operation itself is not difficult, but in dividing the root of the lobe infection of the pleura is inevitable, and all that can be done is to limit its effects. After the age of 35 Lilienthal himself rarely considers that the operation is justifiable, brilliant though its results may be at an earlier age.

A less ambitious but much safer procedure has been devised by Evarts Graham of St. Louis, and as I have carried it out myself with conspicuous success $I$ feel some confidence in the soundness of the method. The first thing is to explore the chest and to see whether or not adhesions are present. If the lung is free adhesions must be produced by artifice, and this is done by packing gauze over the area selected for attack, to be removed at the end of a week. A fortnight later, when the lung has become densely adherent to the chest wall at this point, sufficient ribs are resected over this area to give a really wide exposure of the affected lobe of the lung, a corresponding 
JUNE 5, 1926] H. S. SOUTTAR: RECENT ADVANCES IN THE SURGERY OF THE CHEST. [Mres Trirsh

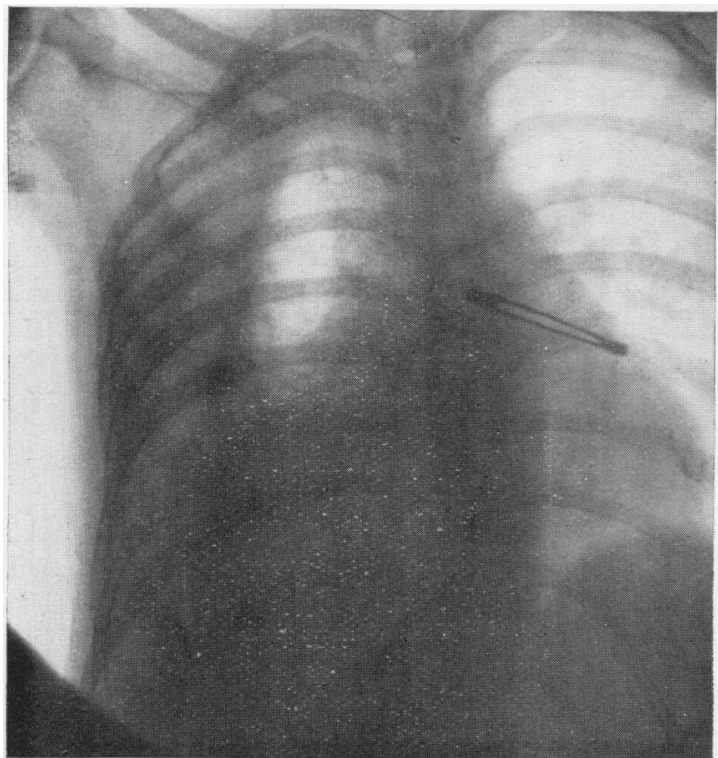

Fig. 1.-Foreign body in right lung; pulmonary abscess.

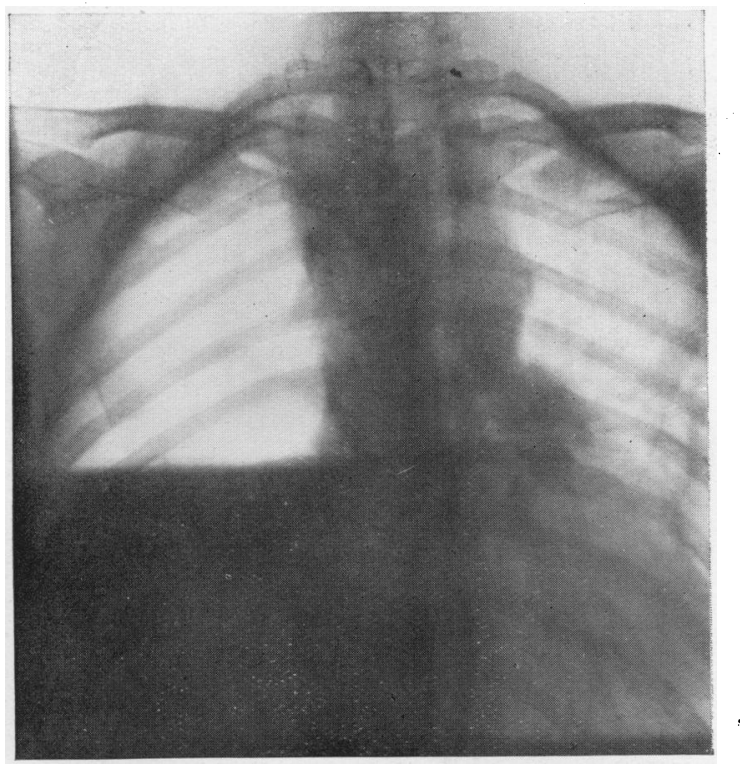

Frg. 3.-Tuberculous empyema; right chest full of fluid.

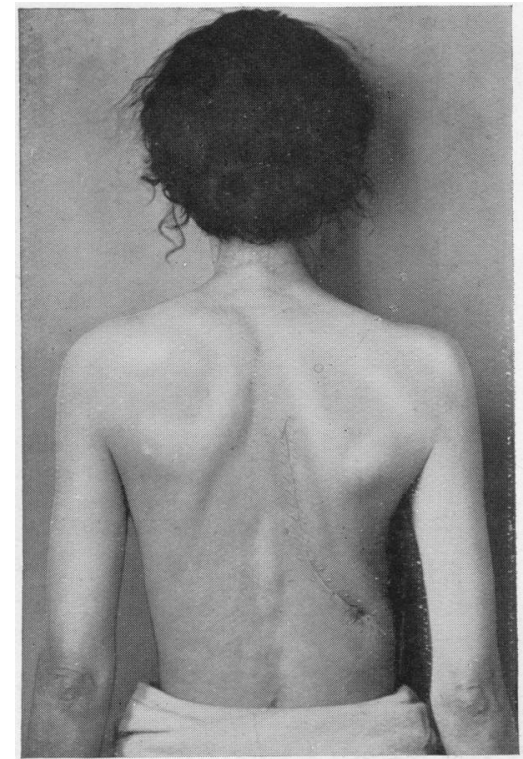

Fig. 3b.-Eighteen months after thoracoplasty for tuberculous empyema of right chest.

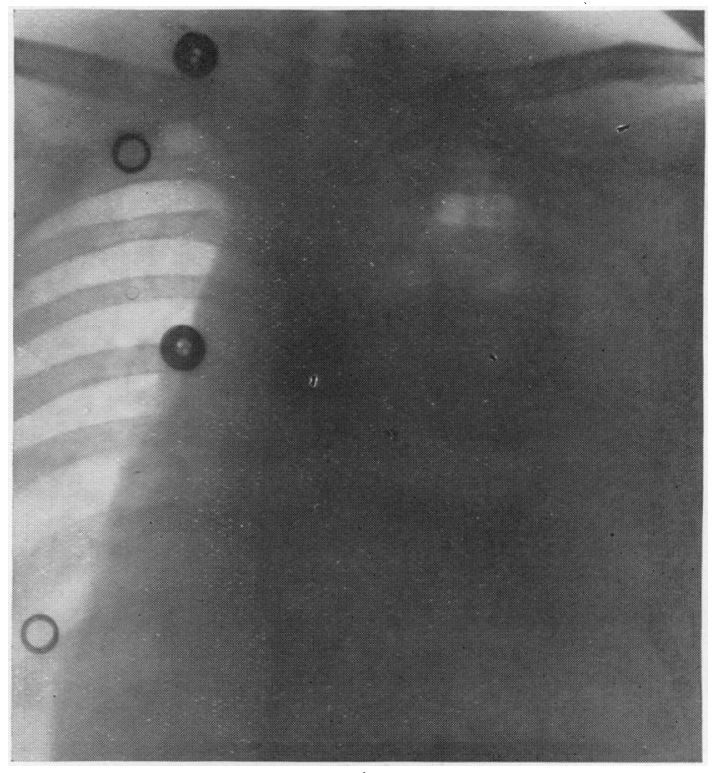

Fig. 2.-Carcinoma of lung; pulmonary abscess.

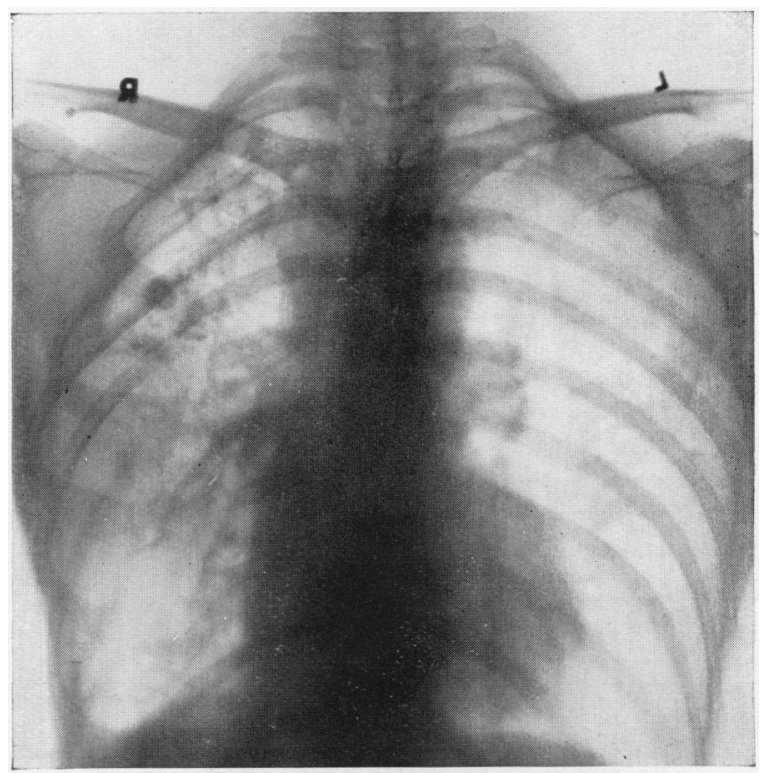

Fig. 3a.-Eighteen months later, after thoracoplasty.

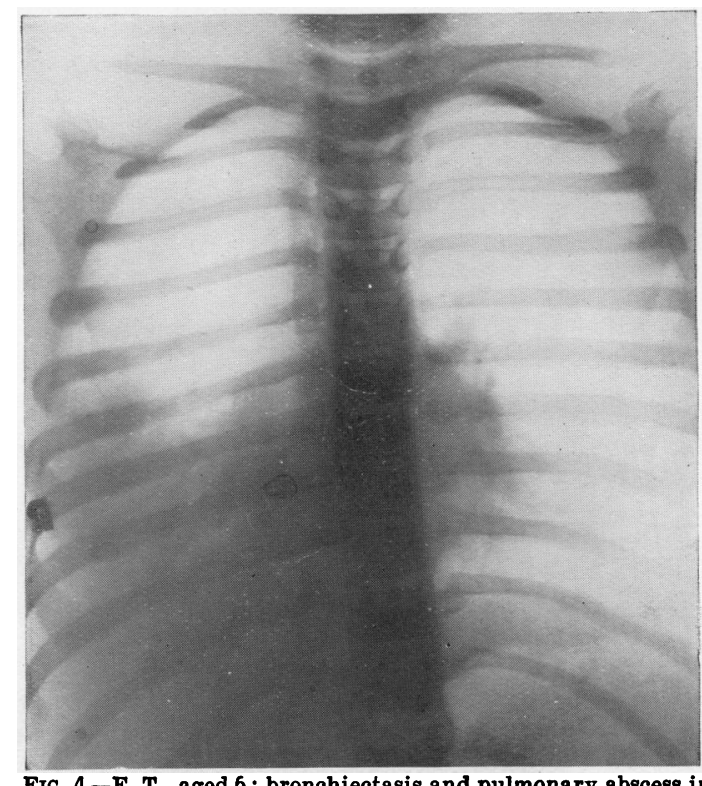

FIG. 4.-E. T., aged 6 ; bronchiectasis and pulmonary abscess in right lower lobe, following tonsillectomy. 
flap of chest wall being turned up. A soldering iron at a dull heat is now plunged straight into the bronchiectatio lung, and the septic spongework is -widely destroyed. A large cavity is left into which open numbers of dilated bronchi, from which pus is thus drained in a really effective manner. A large slough of course comes away, but considerable bleeding is rare, and any that may occur is readily controlled by pressure. The cavity is gradually obliterated by collapse of the chest wall, by expansion of the lung, and by the formation of granulation tissue.

The following case, referred to me by Professor Ellis, illustrates the facility of the operation and the dramatio recovery which may follow.

A woman, aged 31, dated her illness from an attack of pneumonia two years previously which had never quite cleared up. Nine months after this attack she had a slight haemoptysis and was her sputum but she was sent to No tubercle bacill were she remain in bed for four months. For a time last six months she had had a troublesome cough, and an and an evening temperature running up to $102^{\circ}$. She was a thin wasted woman, and said. that she had lost 2 st. in the last six
months. There was marked clubbing of the fingers. Over the months. There was marked clubbing of the fingers. Over the right base there was marked dullness, with diminished vocal resoshowed a dense shadow in this region, suggestive of empyema. She constantly coughed up large quantities of foul pus, of ten blood-stained, and her temperature oscillated between $7^{\circ}$ and $103^{\circ}$.

A needle withdrew streptococcal pus, and an exploration of the chest was therefore carried out. The pleural cavity was found empty and clear of adhesions, except at the point where the needle had been inserted. The right lower point where the impression of being almost solid at its lower pole, which was dark purple in colour. Gauze was packed in between the lung and the chest wall with a view to obtaining adhesions round this area, being withdrawn a week later.

A week later bronchoscopy showed that the right bronchus and the branch leading to the lower lobe were intensely engorged, resembling red velvet, and a cavity of some size was entered; it contained pus. An attempt was made to wash this out, and was repeated on several occasions, but without much success. The trachea was so irritable that bronchoscopy without an anaesthetio led at once to violent coughing and the evacuation of the abscess up the tube. As emptying the abscess had no effect on the temperature it was determined to drain the cavity through the chest wall. A large opening was therefore made over the adherent area, and through this a large cavity in the lung was entered. The opening was enlarged with a heavy copper cautery at a low heat, and by this means the outer wall of a cavity about 3 in. in diameter was destroyed. Into this cavity opened several bronchi as large as a finger, and from these pus poured.

The result was immediate, for her temperature fell at once, her cough stopped, her appetite returned, and she said that she had not been so well for months. After a few days the discharge ceased to be foul and became greatly reduced in amount. She was sent to a convalescent home, and at the end of two months the opening had closed, she had put on a stone in weight, and appeared in perfect health.

Pulmonary Tuberculosis.

To the thoracic surgeon pulmonary tuberculosis presents the widest field of all, and, though its surgical treatment is still in its infancy, in recent years great advances have been made. The underlying principle is that which is universally adopted in the treatment of tuberculosis in other regions-to place the part at rest; but here, in contrast to the limbs, we are dealing with an organ whose movement is essential to life. In the solution of this apparent contraindication lies the whole surgical problem.

The selection of cases is the field of the physician, but perhaps I may refer to a few important points. Of the more acute cases the most suitable are those in which, after two or three months of conservative treatment, the disease is still advancing, and where the advance is more marked on one side. Cases of acute bronchopneumonic or pneumonic disease localized to one lung can be so treated, and the advance may be checked. In chronic cases of long standing with extensive fibrosis and cavitation, where perhaps the disease has come to a standstill, but where the patient is incapacitated by a chronic cough and frequent exacerbations, much can be done for his relief.

The surgical treatment of all these conditions can be summed up in the one word "collapse." It may be obtained by introducing air into the pleural cavity, by introducing packing of some sort between the parietal pleura and the chest wall, by removal of the ribs, or by paralysing the diaphragm, but in every case the final object is the same. Two methods stand out as of very general application, artificial pneumothorax and thoracoplasty, and the two are in many ways complementary to each other. Where it is possible to produce a complete pneumothorax this is undoubtedly the method of choice, but in many cases, and especially in those chronic cases to which we have just referred, adhesions between the layers of the pleura make it impossible, and we may then have to fall back on thoracoplasty.

In producing an artificial pneumothorax any of the standard forms of apparatus may be employed. The essentials are that it should be possible to introduce a known quantity of air and to keep under close observation the resulting intrathoracic pressure. The latter is of extreme importance, since, except in very special circumstances, it is essential that the lung should be allowed to collapse by its own elasticity, whilst as a rule it is best to finish with a small negative pressure. The whole procedure can be carried out under local anaesthesia. The site of puncture should be chosen with a view to avoiding adhesions, and therefore at a distance from the chief foci of disease. In the majority of cases the seventh space in the post-axillary line will be satisfactory. The amount of air introduced on the first occasion will vary between 250 and 450 c.cm., enough to produce definite symptomatic relief, but not enough to run any risk of overstraining the other lung, upon which, of course, additional work is thrown, both circulatory and respiratory. Perhaps two days later more air is introduced, and the operation is repeated at intervals depending on the symptomatic response, until if possible complete collapse of the diseased lung is obtained. If this ideal is reached it will still be necessary to introduce air at intervals of perhaps two weeks, in order to compensato for air which has been absorbed and to maintain the collapse.

Such in briefest outline is a method which has now developed a highly specialized technique. But, like any other operative method, it must only be regarded as an adjuvant to full hygienic control, without which it is doomed to failure. Its success depends upon the possibility of producing a more or less complete pneumothorax - that is to say, upon the absence of adhesions. Whon these are at all extensive other methods must be adopted, and of these the most effective is thoracoplasty.

Thoracoplasty consists in the removal of so much of the bony structure of the thoracic wall that the remaining soft structures collapse upon the underlying lung. The work of Sauerbruch has reduced it from an exceedingly dangerous to a comparatively safe procedure, and in the hands especially of Bull and Saugman it has given brilliant results. Sauerbruch's operation depends upon the fact that if the posterior portions of the ribs are removed the remaining portions can bend inwards on the costal cartilages, and can virtually obliterate the chest cavity. Through a vertical $\mathbf{J}$-shaped incision behind the whole of the posterior half of the thoracic wall is readily exposed, with very little damage to the muscles which cover it. The ribs are exposed in turn and resected subperiosteally, the length removed varying from $3 \mathrm{~cm}$. for the first rib to 12 or $15 \mathrm{~cm}$. for the cighth. The total length of rib removed in a complete opcration may be as much as $120 \mathrm{~cm}$., or about 4 feet. The operation may be limited to one portion of the chest, but it is not then nearly so effective, or it may be done in two stages. It is best accomplished under local and regional infiltration anaesthesia, perhaps accompanied by gas and oxygen. The nerve block is here of the first importance in reducing shock, and with its use the condition of the patient the day after this very extensive operation is quite remarkable.

This is undoubtedly the most powerful method we possess of collapsing the lung, and it is equally applicable to cases where the disease is not in the lung but in the pleura. Of this the following is an example among my own cases.

The patient was a man, aged 25, whom I was asked by Dr. Miller to see in the London Hospital. He gave the histery that five months previously he had noticed a splashing sound in his five months previously found that he was short of breath on chest, and soon after found that he was short of breath on exertion. His doctor found fluid in his chest, and a few weeks fluid were aspirated from the left side of his chest on many occasions, but it always re-collected. It contained tuberclo bacilli. When I first saw him he was a thin, pale man, slightly cranosed, only able to breathe when propped up vertically in bed. cyanosed, only ableting temperature and he was desperately ill The left side of his chest was full of fluid. As it was evident 
that the immediate cause of his trouble was mechanical I had first to consider how this could be relieved. Something more than aspiration through a needle was required, but drainage, from the risk of secondary infection, was out of the question. Under local anaesthesia, and without even moving the patient in bed I made a small opening in the eighth space, into which I fitted a smooth silver obturator as a plug. Through this opening, which remained perfectly clean we removed every morning from which the end of a fortnight his condition had so far improved that I was able to resect a rib and to explor the chest. On opening the pleural cavity twelve pints of milky fluid escaped. Both surfaces of the pleura were covered with tubercles. The wound was closed in the hope that adhesions might form. Six weeks later, fluid having been repeatedly aspirated in the meantime, the whole of the left dorsal region was blocked with novocain, and the posterior portions of the ribs from the fifth to the eighth were resected. As the ninth and tenth had previously been resected satisfactory collapse of the lower part of the chest was thus obtained. The result was very remarkable. He made an uninterrupted recovery, and a year later he was in perfect health, doing full work on a farm. He has remained perfectly well, there is no sign of fluid in his chest, and the external deformity is trivial.

\section{Conolusion}

The thorax thus presents to the surgeon peculiar diffculties and great opportunities. The difficulties are largely mechanical, and they can be overcome by mechanical means. Given the necessary appliances, and the still more necessary experience, there is no region which can be explored with more safety or with greater thoroughness. Many of the conditions have already been brought under our control, and the conquest of many others is only a matter of time. But this of all the branches of our art is the one in which co-operation between physician and surgeon is the most essential, and I therefore welcome this opportunity of bringing it before a body of practitioners who are meeting with these cases every day. I have only touched on a few features in a very large field, but I hope that I may have enabled you to realize the great opportunities, the brilliant hopes, and above all the safety, of modern thoracic surgery.

\section{Ant Addres: \\ ox GASTRIC DIAGNOSIS.* \\ BY}

L. R. BROSTER, O.B.E., D.M., M.CH., F.R.C.S., ASSISTANT SURGEON, CHARING CROSS HOSPITAR; SURGEON, QUEE' HOSPITAL FOR CHILDRER.

(With Special Plate.)

Tris paper contains an analysis of the diagnosis of four related gastric conditions of similar symptomatology, based on operation findings. From the patient's point of view the stomach is the most plaintive organ of the body, and we all know that our methods of diagnosis and treatment of it are not yet so standardized as to meet the requirements of each individual case.

Having been personally interested in the correlation of the $x$-ray diagnosis and the operative findings, I wish to direct attention chiefly to this aspect, and, with the aid of a series of plates supplied by Dr. S. Cochran॰ Shanks, to distinguish the finer points of diagnosis.

I will, so far as possible, limit myself to the analysis of some 140 cases treated at the Charing Cross Hospital; these include 51 cases of gastric ulcer, 33 cases of duodenal ulcer, 27 cases of carcinoma of the stomach, and 26 cases mimicking these lesions, but due to other causes.

\section{Prophylaxis.}

We have not been able as yet to determine the exact causative factor in gastric and duodenal ulcer. There is no doubt that predisposing causes exist. The patient who early seeks medical advice can expect amelioration of his symptoms from judicious treatment, while in the later stages he can be saved much pain and bouts of semiinvalidism, and fear less the complications which threaten his life.

Delivered to the Southampton Division of the British Medica
The practitioner whose aim it is to prevent the occurrence of these lesions has to devise a daily routine for those who seek his guidance. Such questions as diet, the treatment by drugs, the restriction of smoking, proper rest, the elimination of oral sepsis, and of foci of infection must be attended to.

When the disease is established examination by means of the $x$ rays and the fractional test meal should be employed. It is not until such treatment has been tried and has failed to prevent recurrence of the symptoms that the need for surgery arises. It then becomes a question of co-operation between the physician and surgeon, and the best results are obtained when this partnership is continued after the operation. The patient is prone to think that an operation means an end to his disabilities. It is true that most patients are cured, but some are only improved; a few with gross lesions cannot expect to become entirely normal again, while in a small percentage of cases jejunal ulcers will develop. It is to this small minority that continued after-care is so important.

\section{Diagnosir}

With regard to the efficacy of the diagnostic means at our disposal, opinions differ. $1^{N}$-ynihan and the Mayos base most reliance on the $x$-ray findings, Walton stresses the clinical history, while others place their faith in the fractional test meal. The necessity for such indirect methods indicates a very varied symptomatology, and a lack of definite physical signs.

This being so, I am convinced that when taking the patient's history it is necessary to eliminate, as far as possible, matters of fancy, and to consider only the bald facts. For this purpose $I$ use a chart, which is comprehensive and standardizes the results. I prefer to take clinical histories myself, and am often struck by how materially they can differ from those taken by my assistants. In reviewing the $x$-ray and operative findings they are found to agree in the majority of cases, and in this series the margin of error worked out as follows:

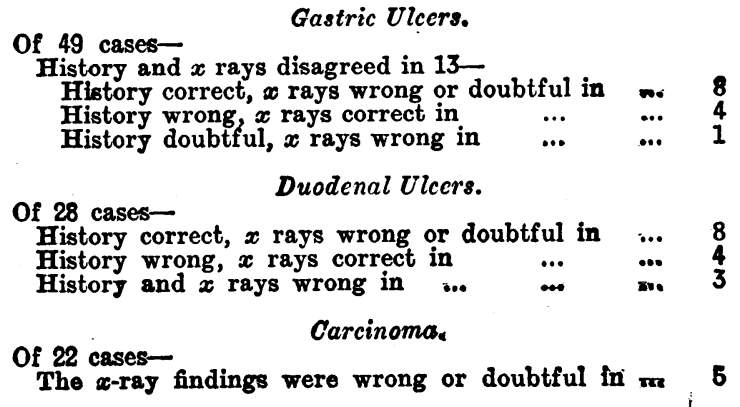

Whatever the merits or demerits of these various forms of investigation may be, the main point is that we each evolve our own particular lines of thought and action, and are best fitted to carry these into effect. Personally I consider a good $x$-ray examination a necessity. Apart from the diagnostic point of view, it is an invaluable guide to the surgeon in planning his operation, and revealing such complications as a small coexisting ulcer. Should there be any doubt, or should the $x$-ray examination and the clinical history disagree, then positive evidence from the test meal becomes a deciding factor.

\section{Symptomatologr.} 1. General.

Perhaps the most striking features of gastric and duodenal ulceration are its selectivity for the male sex, and the definite periodicity of its symptoms. Bouts of pain lasting generally from two to three weeks are followed by intervals of complete freedom ranging from a few months to a year or more. In duodenal ulcer these remissions are most constant; the patient has one or two attacks a year, and knows sometimes exactly when the next one is due.

In the early stages the intervals are not so marked as when the ulcer is established. In the later stages any change in this periodicity becomes significant; the painful attacks become prolonged, while the periods of freedom 